\title{
APPARATUS FOR THE ESTIMATION OF FREE AND ALBUMINOID AMMONIA IN WATER ANALYSIS.
}

By Augustus H. Gill, Ph.D.

THE article by Mr. Embrey* upon this same subject, has induced me to describe the apparatus for these determinations in use in the laboratory for water analysis of the Massachusetts Institute of Technology, used by the State Board of Health.

The arrangement will, I think, be evident from the accompanying sketch. The apparatus consists of a nearly spherical flask with square shoulders, of $850-900$ c.c. capacity, connected by a bent glass tube and "cork joint" with a vertical block tin condenser. The "cork joint" is here shown in section, and consists of a sound cork fitted at one end with the condenser, and at the other, with the glass tube, indicated by the cross hatching, which enters the former for about an inch-and-a-half, thus avoiding all contact of steam with the cork, and making a

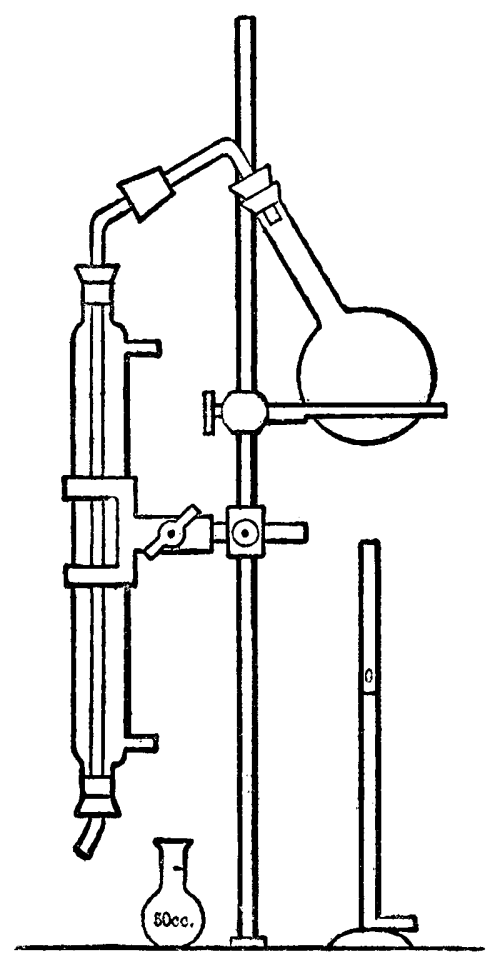

* The Analyst, xvii., 41. 
tight and durable connection; the flask is closed with a superfire curk carrying the bent glass tube.

The condenser is the usual glass jacket, with the inside tube of block tin, three ounces to the foot, one-quarter inch internal diameter, twenty-four inches long and bent at the top for the "cork joint," and at the bottom for delivery into the fifty c.c. graduated flasks. $\uparrow$ The lamps used are ordinary Bunsen burners lengthened by inserting a piece of pipe between the base and the burner proper. The whole apparatus is supported by the ring and clamp upon a brass rod fixed into the distilling table. This table is eighteen

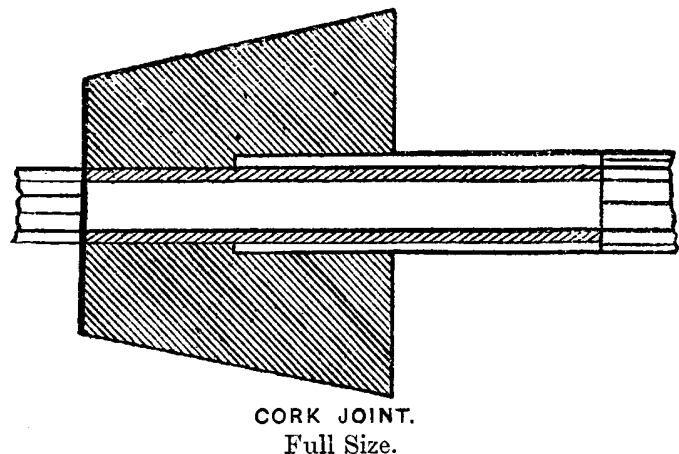

feet long, two feet wide, and three feet high, with two thirteen-inch sinks near each end; upon it are arranged the two rows of brass rods five inches apart, with eighteen inches between each rod, thus affording space for fourteen sets of apparatus, and making the laboratory equipment capable of handling sixty to seventy samples per day. All the piping is underneath the table, the rubber tubing passing through it, one set of tubing being painted black to distinguish the rows; each condenser and lamp is provided with its own stopcock, or the whole can be turned off together.

For designating the number of the water analyzed, slips of ground glass are placed between the vertical rods.

The apparatus is distilled free from ammonia before each determination, and, as we have waters that show neither free nor albuminoid ammonia, it indicates that the working is satisfactory.

The condensation, even in the hottest weather, is absolutely perfect. Spiral condensers of glass and tin have been used, but were too fragile and cumbersome to admit of their continuance.

With this arrangement one person performs fourteen distillations simultaneously in about an hour, the work all being done from one side. The present form has been in use eighteen months, during which time about 5,000 determinations have been made, which is sufficient proof of its practicability.

† Flasks are here used, as the Nessler tubes are inconvenient to manare ; those in use hold 50 c.c. are 8 inches long and $\frac{5}{8}$ inch internal diameter, admitting of the readiug of $0.000001 \mathrm{gm}$. of armmonia, with absolute certainty. 
Observations on the methods in use for the determination of the quantity of gas dissolved in potable waters. G. Musaio. (Staz. Sper. Ag. Ital. xxiii. 113.) - In the determination of the amount of gas dissolved in watel there are several sources of error, which arise from the entranee of water into the vessel in which the gas is measured; these are the solubility of the gas in the small quantity of water in the tube, and the oxidation on the mercury in the presence of water. Another source of error in the determination of oxygen lies in the absorption by pyrogallate of potash; either the absorption is not complete, or carbonic anhydride is liberated and measured with the unabsorbed gas. To avoid these errors the author proposes a new form of apparatus; this consists of three flasks arranged as shown in fig. 1. The flask A contains the water

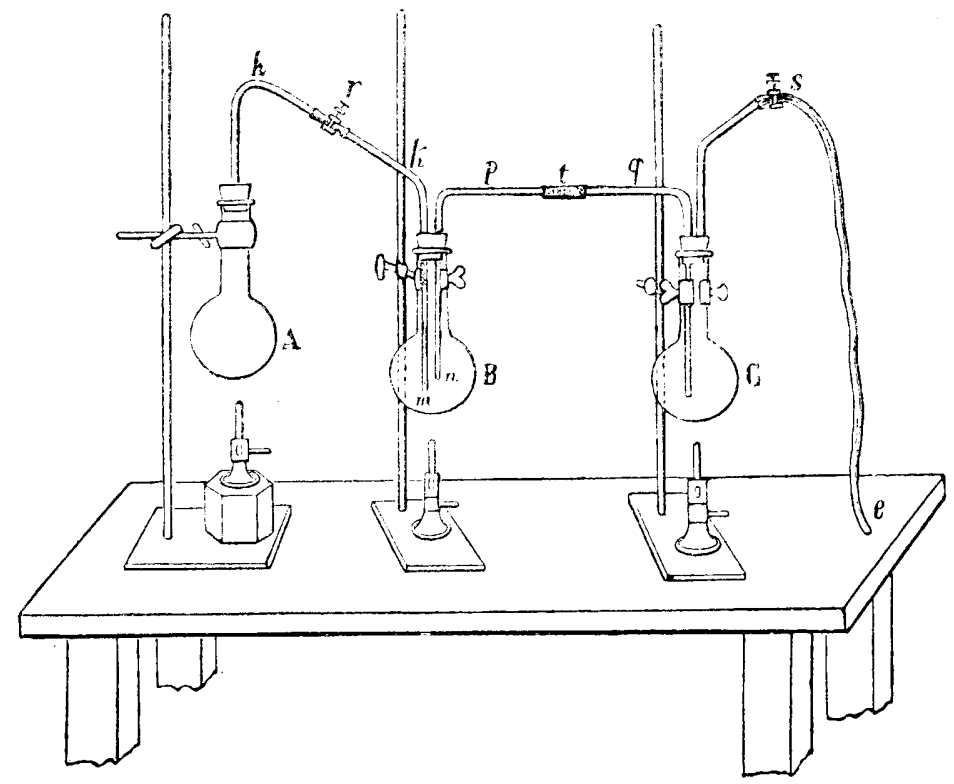

under examination, and is closed by an indiarubber cork, through which passes the tube $\mathrm{H}$; the extremity of this tube is drawn out, and is attached to the rest of the apparatus by an indiarubber tube, on which is a screw pinchcock. The flask can thus be filled and placed in position without risk of the water escaping.

The flask $B$ acts as gasholder; it is closed by an indiarubber cork through which pass two tubes, as shown in figure. The obtuse-angled tube is divided in two parts, which are joined by an indiarubber tube just after passing through the cork. When joined the tube passes almost to the bottom of the fjask; but if the upper portion is carefully withdrawn, the lower portion drops off, so that any gas in the flask may be completely expelled by the upper portion. The third flask is simply used as a reservoir. The apparatus is used as follows :-The flask $\mathrm{B}$ and $\mathrm{C}$ are nearly filled with distilled water, 
and the corks are simply placed in them so as to allow steam to escape; the water in them is boiled for from 35 to 40 minutes; then the cork of $\mathrm{C}$ is inserted, and air is blown in till $\mathrm{B}$ is completely filled. The cork of $\mathrm{B}$ is next inserted, and this flask is then quite full. The two pinchcocks $\mathbf{R}$ and $\mathbf{S}$ are closed.

The flask $\mathbf{A}$ is filled with the water under examination; the pinchcocks are slightly opened, and air is blown in. When a small stream of hot water issues from the tube the drawn-out end of the tube $\mathrm{H}$ is inserted therein, and the apparatus is ready. The gas is lighter under the flask $\mathrm{A}$, and under the flasks $\mathrm{B}$ and $\mathrm{C}$ when necessary to keep the water $\mathrm{n}$ them hot. The gas passes into $\mathrm{B}$, and the displaced water flows into C. After forty minutes to an hour's boiling the pinchcock $R$ is closed and the tube $H$ withdrawn. The tube $\mathrm{K}$ is gently raised till the part $\mathrm{D} \mathbf{M}$ drops off. The tube $\mathbf{K}$ can be emptied of water by allowing a flame to play on it for a second or two. A tube of small bore is inserted into the indiarubber tube, and the gas transferred through it into a measuring tube standing over mercury by blowing through the tube $\mathrm{E}$ till the water reaches the pinchcock R. No correction need be made for the small quantity of air in the small borer tube.

The analysis is performed by first pushing into the tube a piece of caustic potash, and noting the absorption. This is called carbonic anhydride. The author prefers pyrogallate of potash to cuprous chloride, sodium hydrosulphite, or ferrous sulphate and potash, as an absorbent for oxygen. He pushes up a ball of papier-mâché soaked in pyrogallate of potash, and repeats the treatment until no more is absorbed. He then uses a piece of caustic potash to absorb any carbonic anhydride formed in the reaction between oxygen and the pyrogallate. The residual gas is taken as nitrogen. All gases are reduced to the standard pressure and temperature. The remainder of this paper is devoted to the discussion of a few results obtained with saturated distilled water, and the water of his laboratory. In the latter the results for oxygen and nitrogen were those required by theory for a fully-saturated water, but the carbonic anhydride was notably deficient of the amount required to form bi-carbonates.

H. D. R.

Estimation of Oxygen in Lead. G. Lunge \& E. Schmid. (Zeit. f. Anorg. Chem. 1892, II., 451 to 460.) - The shortness of re-melted lead has been attributed to the presence of dissolved oxide, and it has been stated that the resistance of lead to sulphuric acid depends on its content of oxygen. The authors therefore deem the determination of oxygen in lead a matter of some little importance. Their method is to pass hydrogen through the molten metal and weigh the water produced. The hydrogen from a Kipp's apparatus is passed through a wash-bottle containing caustic soda, one containing a solution of lead oxide in caustic potash (to absorb $\mathrm{H}_{2} \mathrm{~S}$ ), two 
containing silver nitrate solution, and two containing strong sulphuric acid; the gas then passes through a tube containing platinised asbestos, and heated in a furnace; this is followed by a wash-bottle of sulphuric acid and two $U$ tubes containing phosphorus pentoxide. The hydrogen thus purified from the last traces of oxygen, is then passed through the molten lead contained in a hard glass tube:-

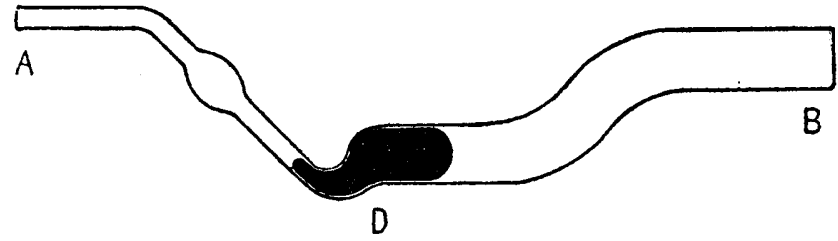

The hydrogen passes in at the end $A$, the metal is kept molten at D by a fan-shaped bunsen burner, and the water is caught by a phosphorus pentoxide $U$ tube attached by means of a cork at B. A guard wash bottle of sulphuric acid completes the apparatus. The hydrogen is allowed to pass for two hours to free the apparatus from oxygen, and for another two hours after the platinised asbestos has been heated to redness. The authors made several experiments to prove the purity of the hydrogen at the end of the four hours, and are satisfied that this time is sufficient to ensure the absence of oxygen. The quantity of lead used should be under 30 grammes, and it, of course, must not be melted until the apparatus is absolutely free from oxygen. Half-an-hour is said to be sufficient to deoxidise the lead. The final $U$ tube is then weighed with all the usual precautions.

The following figures may be quoted from the authors' tabulated results :-

\begin{tabular}{|c|c|c|c|c|c|}
\hline & & & & & $\begin{array}{l}\text { Oxygen } \\
\text { Per Cent. }\end{array}$ \\
\hline Firsts (Jungfernblei) & $\ldots$ & $\ldots$ & $\ldots$ & $\ldots$ & 0.00237 \\
\hline Do. alloyed with 0 . & 2 per cent. o & f antil & mony & $\ldots$ & 0.00363 \\
\hline Refined lead, alloyed & with 0.02 per & cent. & of antimo & my & $0 \cdot 00250$ \\
\hline Do. & $0 \cdot 1$ & " & of copper & ... & $0 \cdot 00343$ \\
\hline Do. & 0.2 & ", & " & $\ldots$ & 0.00566 \\
\hline Do. & $1 \cdot 0$ & , & , & $\ldots$ & 0.03661 \\
\hline
\end{tabular}

Attention is called to the influence of copper in increasing the dissolved oxygen.

A. G. B.

Sulphurous Acid in Wine. M. Ripper. (J. Prakt. Chem. [2], xlvi., 428473.)--The author has made a searching investigation into the condition in which sulphurous acid exists in wines, and into the methods for its estimation; his experiments are fully detailed in the paper, which is of considerable interest. 
Haas' method (Berichte, 1882, xv., 154), for the estimation in question consists in distilling the wine in a suitable apparatus, through which a current of carbonic dioxide is passed, and collecting the distillate in a bulbed $U$ tube containing iodine solution; the sulphurous acid is thus oxidised to sulphuric acid which is weighed as barium sulphate. Desiring to use this as a standard process, the author sought for possible errors in it and alighted upon oxygen and hydrogen sulphide in the carbon dioxide as likely to influence the results ; he states that the former gas is always present in carbon dioxide, made from marble and hydrochloric acid, and that the latter may in many cases be detected. These sources of error would tend to neutralise each other had they any real existence; that they have can hardly be allowed when the numbers obtained with carbon dioxide washed by water only, and by potassium permanganate and chromous chloride are compared. A more real source of error was found to exist in the impurity of the barium sulphate, upon which point the author has already written. (The Anaryst, 1892, 233).

As an analytical process Haas' method is too lengthy and the author modifies it by collecting the distillate in caustic potash, acidifying this solution and titrating it with $\mathrm{N} / 50$ iodine solution. The details are as follows :- 50 c.c. of the wine are pipetted into a distillation apparatus through which a current of hydrogen is passing. 5 c.c. of sulphuric acid $(1: 3)$ are added and the flask is heated in a glycerine bath at $80^{\circ}-85^{\circ}$ for three-quarters of an hour, the current of hydrogen being maintained the while. The sulphurous acid is absorbed by 20 c.c. of $\mathrm{N}$-potash in the receiver, and after the time stated is liberated by the addition of 10 c.c. of sulphuric acid $(1: 3)$ and titrated with $\mathrm{N} / 50$ iodine solution, starch being used as an indicator. A number of experiments are quoted to show that no other easily oxidisable, volatile constituent of the wine passes over with the sulphurous acid Experiments were then made to ascertain whether the direct titration of the sulphurous acid in a white wine be possible. With regard to red wines it may be said that they seldom contain sulphurous acid, inasmuch as their colour would be damaged by sulphuring ; should an estimation be necessary it must be made by the distillation process. The fact that the iodine consumed by an acidified white wine is far below that necessary to oxidise the sulphurous acid present, as estimated by the distillation method soon brought the author to the conclusion that the sulphurous acid in wine is not all present as free acid or as alkaline sulphite. After many experiments it was found that aldehyde sulphite is the other form in which the sulphurous acid exists in the wine; this is not immediately oxidisable by iodine in presence of sulphuric acid, but as might be expected, is split up by the acid during distillation: it is, however, noteworthy, that the aldehyde and sulphurous acid in the distillate will again combine after a short time if no substance be present to remove one or the other. Very brief contact with caustic potash will decompose the aldehyde sulphite, and this opens the door for the determination of the total sulphurous acid in wine by direct titration. To this end 50 c.c. of the wine are pipetted into a 200 c.c. flask containing 25 c.c. of normal potash, the nose of the pipette being inserted into the liquid; after some $10-15$ minutes 10 c,c. of sulphuric acid 
$(1: 3)$ are added, and titration with $\mathrm{N} / 50$ iodine solution effected, starch being added as indicator. The free sulphurous acid is then determined by pipetting 50 c.c. of the wine into a flask which has had a stream of carbon dioxide passed through it, adding 5 c.c. of sulphuric acid ( $1: 3$ ), and titrating at once with the $\mathrm{N} / 50$ iodine until the blue colour is permanent for a short time, after which the tannins, etc., in the wine begin to beoxidised. The difference between the total and the "free" sulphurous acid (i.e., that existing as such and as alkaline sulphites), is termed "aldehyde" sulphurous acid by the author. By adding sulphurous acid to unsulphured wines, he showed that when the wine is kept the free sulphurous acid decreases and the aldehyde sulphurous acid increases, while the total sulphurous acid also decreases.

The author gives no summary as to the quantity of sulphurous acid in wines, but from the figures he quotes it would seem to average a little over 0.01 gram. per 100 c.c of the Rhine wines which he analysed.

A. G. B. 